\title{
Treating Women With Urinary Incontinence and Overactive Bladder
}

Nurul Yaqin Mohd Nor ${ }^{1}$, Zalina Nusee ${ }^{1}$

${ }^{1}$ Department of Obstetric and Gynaecology, Kulliyyah of Medicine, International Islamic University Malaysia

Presenter: Nurul Yaqin Mohd Nor

Introduction: Pelvic floor Muscle Training (PFMT) commonly used physical therapy for Female Overactive bladder (OAB) and stress urinary incontinence (SUI). However some women has difficulty to understand and comply to PFMT. Our aim is to evaluate the effectiveness of Transpelviner Magnetic Stimulation (TPMS) as a replacement for PFMT. Materials and Methods: A prospective observational study on 53 women with SUI or OAB, were assessed objectively using cough stress test, bladder diary, one hour pad test, perineometry and validated questionnaires. Then, all of them received 10 courses of individualized TPMS therapy over five weeks. Reassessments were done after five weeks and six months of initial treatment. The outcomes were measured by severity index, negative pad test, muscle power, numbers of leaking episodes, daytime frequency and nocturia, negative cough stress test and improvement in specific quality of life questionnaires (QOL) Results: Frequency of micturition in $\mathrm{OAB}$ showed reduction from $100 \%$ at baseline to $12.5 \%,(p=0.053)$ after five weeks and $25 \%,(p=0.345)$ after six months. While, SUI reducedfrom $100 \%$ to $17.2(0.052)$ and $27.6 \%(p=0.826)$ after six months. Urgency OAB reduced from $100 \%$ to $12.5 \%$ and SUI reduced from $100 \%$ to $41.4 \%$. It appears that after five weeks of treatment, all participants improved in their severity index (75\%) followed by OAB (55.6\%). However the percentage dropped six months later which was $50 \%$ and $12.5 \%$, respectively. These results were consistent for perineal muscles power, one hour pad test and QOL score. Conclusion: TPMS shows significant effectiveness to treat OAB and SUI. However, bigger study is required to compare it with PFMT in treating urinary symptoms. 